\title{
Aortic Valve Replacement by Pulmonary Autogrbft: Technical Advances and Follow-up
}

\author{
M Aftabuddin', OS Khan'1, AA Rama², C Acar², A Pavee², I Grandjbakhch² \\ ${ }^{1}$ Department of Cardiac Surgery, BSMMU, Dhaka. ${ }^{2}$ Department of Cardiothoracic Surgry, Lapitie \\ Salpiteriere, Paris.
}

KeywordsAortic valve, Pulmonary Autograft, Ross procedure.

\begin{abstract}
:
Background: Autograft is the best choice of graft material in every place especially in cardiac surgery to avoid all type of anti-coagulant related hazard, immunological reactions and considering cost involvement. Study was designed on the basis of above facts.

Methods: All patients underwent surgery with standard Cardiopulmonary By-pass under moderate hypothermia using median stemotomy incision. Aortic valve was excised and the right and left coronary buttons were fashioned. Also the pulmonary root was excised. A running suture technique was used to insert the autograft in to the aortic annulus. A pulmonary homograft made by pericardium with three pulmonary valves of appropriate size $(22-29 \mathrm{~mm})$ was then inserted.
\end{abstract}

Results: Between July 2001 to July 2002, 30 patients underwent the modified Ross Procedure at LaPitie Salpitriere Hospital, Paris, France. Out of 30 patients 16 were male and 14 were female with the mean age of 29 years. Indication of operation was aortic stenosis in 6 cases, aortic insufficiency in 9 cases and mixed aortic valve lesion in 15 cases. The mean intra operative cross clamp time was 120 minutes (range: 70-170 minutes), mean cardiopulmonary bypass time was 150 minutes (range: 110195 minutes). Postoperativee chocardiogramsr evealed no significant pulmonary or aortic valvular dysfunction. Three patients, early in the series required reopening for post operative bleeding. Overall a mean of 2.5 units of bloods (0-10) were transfused per patient. Atrial fibrillation occurred in 2 patients which wsre managed accordingly. Among 30 patients, there was one perioperative death (3\%), one patient was lost to follow-up and one patient refused to late post operative echocardiography. Twenty four caseso ut of the twenty seven patients available for functional follow-up were in NYHA class I, 2 patients in NYHA class II and one patient in NYHA class III.

Conclusions: Pulmonary autografts in aortic position give good short-term result but for its long \{erm effrcacy and safety needs more study.

(Cardiovasc.j. 2011; 4(1): 58-60)

\section{Introduction:}

Replacement of the aortic valve with a pulmonary autograft and insertion of a pulmonary or aortic adults. ${ }^{1,2,3}$ Some modification of the original technique have now been standardized. ${ }^{4} \mathrm{We}$ described our technical modification with minimal bleeding, graft failure and good clinical follow-up finding homograft in the right venhicular outflow tract using Ross procedure was described by Ronald Ross. ${ }^{1}$ Pulmonary autograft has the capability to provide long-term valve competence for many years without any anticoagulant and also allow normal growth. Ross procedure has been established as an effective method of aortic valve replacement (AVR) with good result in children and young.

\section{Patients and Methods:}

Between July 2001 to July 2002, 30 patients underwent the modified Ross Procedure at La-Pitie Salpitriere Hospital, Paris, France. Out of 30 patients 16 were male and 14 were female with the mean age of 29 years. Age was ranging from 16 to 42 years.Indication of operation was aortic stenosis in 6 cases, aortic insufficiency in 9 cases and mixed aortic valve lesion in 15 cases. In 12 cases the lesion was congenital, 5 had endocarditis and 13 had rheumatic origin.

\section{Surgical Technique}

All patients underwent surgery with standard Cardiopulmonary By-pass under moderate hypothermia using median sternotomy incision. Aortic root replacement technique was used in all

Address of Correspondence: Prof. Mohammad Aftabuddin, Chairman, Department of Cardiac Surgery, Bangbandhu Sheikh Mujib Medical University, Dhaka, Bangladesh. E- mail: aftab12uddin@yahoo.com. 
patients. Aortic valve was excised via a transverse aortic incision just above the commisures, and the right and left coronary buttons were fashioned. The pulmonary root was excised distally just proximal to the pulmonary arterial bifurcation, and proximally leaving a $3 \mathrm{~mm}$ margin below the lowest portion of the pulmonary valve leaflets. A running suture technique was used to insert the autograft in to the aortic annulus and study should be continued. The buttons were anastomosed using running 6-0 polypropylene.The distal aortic anastomosis was constructed with running 4-0 polypropylene. A pulmonary homograft made by pericardium with three pulmonary valves of appropriate size (22-29 $\mathrm{mm}$ ) was then inserted.The distal anastomosis was made at the widest point of the pulmonary artery at approximately near the bifurcation, using running 3-0 polypropylene proximally and 4-0 polypropylene distally to replace the right ventricular out flow tract.

\section{Follow-up}

Postoperative follow-up data were obtained by regular clinical follow-up and re-examination of the patients. All patients underwent preoperative and postoperative M-mode, two dimensional, colour flow \& and Doppler echocardiography before discharge. Follow-up echocardiography were obtained two months interval. NYHA functional class assessment was performed two month interval.

\section{Results:}

The mean intra operative cross clamp time was 120 minutes (range:70- I70 minutes), mean cardiopulmonary bypass time was 150 minutes (range: 110-195 minutes). Postoperative echocardiograms were obtained in the operating room and before discharge from the hospital. All revealed no significant pulmonary or aortic valvular dysfunction .Three patients, early in the series required reopening for post operative bleeding. Bleeding sites were at the proximal suture line of the autograft in aortic position in one patient, in the proximal suture line of the pulmonary homograft in right ventricular out flow in one patient and at the multiple sites in one patient (due to coagulopathy). Overall a mean of 2.5 units of bloods (0-10) were transfused per patient. Other complications included atial fibrillation in 2 patients which were managed.

\section{Post- operative Follow-up}

Among 30 patients, there was one peri -operative death (3\%), one patient was lost to follow-up and one patient refused to late post operative echocardiography. Thus, 27 patients were available for follow-up analysis. The pulmonary auto graft root diameter ranged from $22-40 \mathrm{~mm}$ and dilated more than $36 \mathrm{~mm}$ in three patients. Among these three patients, one had severe aortic insufficiency (AI), one had moderate AI and one had only trace AI. It is interesting that all 3 patients who suffered aortic root dilatation had original aortic bicuspid valve. One patient with normal aortic root diameter also had mild AI. The homograft pulmonary valve was visualizedi $n$ the follow -up echoc ardiograms. There was mild insufficiency in 2 patients. In addition, there was mild stenosis in 2 patients and severe stenosis (peak gradient 75 $\mathrm{mm} \mathrm{Hg}$ ) in one patient. All patient with moderate stenosis remained in NYHA class I. Over all, 24 cases out of the 27 patients available for functional follow-up were in NYHA class I, 2 patients in NYHA class II and one patient in NYHA class III.

\section{Discussion:}

Choice of the aortic valve conduit for replacementin the young patients include bioprosthesis, mechanical valves, cryo preserved homografts and auto graft operation using pulmonary valve. Bioprosthetic valves have a low incidence of thromboembolism, degenerate rapidly in younger patients. Mechanical valves have excellent durability, but require lifelong anticoagulation. Homograft in aortic position has a low incidence of thromboembolism and low incidence of reoperation, but have accelerated rate of degeneration in young adult. Pulmonary autograft to the aortic position is $100 \%$ vital and provides a growing valve in children. Recently it is confirmed that, after implantation, cryo preserved homografts do not retain viable cell, while transplanted pulmonary valves display near nonnal architecture and cellularity.

Incidence of post operative bleeding was $8 \%$, perioperative myocardial infarction $4 \%, 4 \%$ incidence of stroke and $3 \%$ perioperative mortality in one study. ${ }^{8}$ The majority of perioperative bleeding occurs at the proximal anastomosis of either the pulmonary autograft or homograft, which happened in two patients in our series. This 
can be minimized by incorporating a Teflon felt strip around the suture line or by simply suturing the residual aorta to the everted edge of the ventricular muscle with the autograft using running 4-0 prolene. Other complication of this procedure such as endocarditis, valve failure was less than different studies. ${ }^{3,4,5}$ Some authors suggest that a mismatch between the aortic root diameter and auto transplanted pulmonary root is responsible for progressive dilatation and eventual failure of the pulmonary autograft. Reduction of aortic annulus before graft insertion or using a Teflon felt may prevent progressive aortic root dilatation or valve insufficiency. Intrinsic connective tissue diseases in the active pulmonary auto graft in patients with bicuspid aortic valves may contribute to progressive dilatation of the pulmonary autograft root and concomitant insufficiency. Data in our series reveals a higher association of pulmonary aortic root dilatation in patients whose original valve was bicuspid. Development of pulmonary homograft stenosis is another cause of late re-operation following the Ross Procedure. In our series, a significant number of patients have developed pulmonary stenosis which is consistent with others. ${ }^{8}$ Technical factors, autoimmune destruction may cause stricture development but this does not occur in the auto transplanted valve. Balloon dilatation may allow for non-surgical treatment of the pulmonary stenosis after this procedure.

In conclusion, pulmonary autograft in aortic position give good short-term result but for its long -term effrcacy and safety needs more study.

\section{References:}

1. Bachet K, TermigunJ L, Goudol B , et al. Aortic root replacement with a composite graft. Factor influencing immediate and long term result. Eur J Cardiovasc Surg 1996; 10:207-13.

2. Bohm JO, Hotha CA, Hemmer WB, et al. Older patient fare result with the Ross procedure Ann Thorac surg 2003; 75:769-807.

3. Chamber CC, Sommervillie J, Stone S, et al. Fulmonary autograft proceduref or aortic valve diseases. Long term result of the pionner's series. Circulation 1997: 96: 220614.

4. Davi TE. Surgery of the aortic valve. Curr probl surg 1999; 36: 421- 542.

5. Ekim BC. The Ross operation:a 13 years experience. Ann Thorac Surg 1999; 86: 814-30.

6. Gerosa G, M kay B, Ross DN. Replacement of aortic root or aortic valve with pulmonary allograft. Ann Thorac surg 1991; 51: 424- 9.

7. Gott Vl, Greene PS, Alijo DL, et al. Replacement of aortic in Marfan's syndrome. New Eng J Med 1999; 340: 1307- 13 .

8. Hirose Y, Hamada S, Takamia M, et al. Aortic aneurysms growth rates measured in CT. Radiology 1992; 185: 249-52. 